\title{
NUEVOS REGISTROS DE PSYCHODIDAE (DIPTERA), CON UNA LISTA DE LAS ESPECIES CITADAS PARA CHILE
}

\author{
NEW RECORDS OF PSYCHODIDAE (DIPTERA) \\ WITH A LIST OF THE SPECIES CITED FOR CHILE
}

Mario Elgueta ${ }^{1} \&$ Jan Ježek ${ }^{2}$

\begin{abstract}
RESUMEN
Se entrega una lista de cuarenta especies citadas para Chile, incluyendo nuevos registros; en el caso de nueve especies se incluyen distribuciones basadas en material depositado en la Colección Nacional de Diptera del Museo Nacional de Historia Natural (Santiago, Chile).

Se establecen las siguientes nuevas combinaciones: Psychodocha pseudocompar (Tonnoir, 1929) comb. nov.; Psychodocha simillima (Tonnoir, 1929) comb. nov.; Desmioza equalis (Tonnoir, 1934) comb. nov.

Se discute sobre la distribución geográfica y validez de algunas especies y se agregan las referencias que respaldan los aspectos taxonómicos, biológicos y de distribución geográfica de cada especie.

Palabras clave: Lista de especies, nuevas combinaciones, distribución geográfica.

\section{ABSTRACT}

A list of fourthy species cited from Chile is given and new records are included; for nine species distributions are based on specimens deposited in the National Diptera Collection from the Museo Nacional de Historia Natural (Santiago, Chile).

New combinations are established: Psychodocha pseudocompar (Tonnoir, 1929) comb. nov.; Psychodocha simillima (Tonnoir, 1929) comb. nov.; Desmioza equalis (Tonnoir, 1934) comb. nov.

1 Área de Entomología, Museo Nacional de Historia Natural, Casilla 787, Santiago (Correo central), Chile. mario.elgueta@ mnhn.cl

2 Department of Entomology, National Museum, Kunratice 1, CZ-148 00 Praha 4, Czech Republic. jan.jezek@quick.cz
\end{abstract}


The synonymy and validity of some species are discussed, suitable and important references are added. Distribution of all included species and some biological data is given.

Key words: Species list, new combinations, geographic distribution.

\section{INTRODUCCIÓN}

Los adultos de la familia Psychodidae son conocidos como moscas polilla, moscas de la arena o moscas búho; este último nombre seguramente hace referencia a su periodo de actividad, que en la mayoría de los casos es nocturno. Se presentan comúnmente en ambientes húmedos y sombríos y algunas especies se presentan también en lugares habitados, al interior de casas y otras edificaciones. En algunos grupos se presentan larvas saprófagas asociadas a materia orgánica en descomposición, otras se asocian a cursos de agua $e$ incluso algunas se encuentran en zonas áridas, desarrollándose en madrigueras de mamíferos.

En relación a la alimentación de adultos, se ha indicado que se alimentan de materiales azucarados o materia en descomposición; también hay un importante grupo de hematófagos, integrantes de la subfamilia Phlebotominae, de importancia en salud humana (Foote, 1991). Respecto de este último grupo, existen casos de miasis reportados para América del Sur (DonosoBarros \& Cambiazzo, 1950; Mariluis et al. 2007). Hasta hace muy poco tiempo no se había registrado representantes de Phlebotominae para Chile (Young \& Duncan, 1994) y sólo se tenía una mención relativa a la identificación por parte del Dr. Guillermo Kuschel, en 1951, del primer flebótomo de Chile (Le Pont et al. 1998); esta primera identificación fue hecha sobre una hembra recolectada en Algarrobo, la que con posterioridad resultó corresponder a Lutzomyia isopsi Léger \& Ferte ( $\mathrm{F}$. Le Pont comunicación personal).

Respecto de la representación de esta familia en Chile, Stuardo (1946) señalaba la presencia de 4 géneros y 20 especies; más recientemente Duckhouse (1973) registra 4 géneros con un total de 25 especies y recientemente González (2013) repite la cifra de 4 géneros pero disminuye la cantidad de especies a 15; en este último caso seguramente no se incluye en el recuento a algunos géneros y especies de Psychodinae tratados por Quate \& Brown (2006), otras especies descritas antiguamente y que hasta ahora no ha sido posible reconocer y tampoco a Nemopalpus stuckenbergi Wagner, 2012.

A nivel mundial se reconocen seis subfamilias de Psychodidae y sólo Horaiellinae no se encuentra representada en Chile; las restantes subfamilias corresponden a Bruchomyiinae (Nemopalpus), Psychodinae (Desmioza, Didicrum, Logima, Nemoneura, Psychoda, Psychodocha y Tinearia), Phlebotominae (Lutzomyia), Sycoracinae (Aposycorax) y Trichomyiinae (Trichomyia).

En el caso de América del Sur recientemente se han desarrollado investigaciones en diferentes países, cuyos resultados pueden servir de referencia para otros estudios (Cordeiro et al. 2013; MoyaArévalo et al. 2012; Omad, 2014; Omad \& Rossi, 2012).

El propósito del presente aporte es entregar las identificaciones de ejemplares de la colección del Museo Nacional de Historia Natural, Chile. Se entrega también una lista de las especies que han sido citadas para Chile, incluyendo distribución geográfica conocida; para cada una de ellas, se detallan todas las referencias que avalan los datos entregados.

\section{MATERIALES Y MÉTODOS}

Se estudian e identifican ejemplares mantenidos en seco, provenientes de distintas localidades de Chile y recolectados en gran parte por el Dr. Guillermo Kuschel (Auckland, Nueva Zelanda), los que se encuentran depositados en la Colección Nacional de Insectos del Museo Nacional de Historia Natural, Santiago, Chile (MNHNCL).

Para la correcta identificación de los ejemplares fue necesario efectuar preparaciones en portaobjetos y utilizando las técnicas habituales; los ejemplares secos fueron mantenidos por breve tiempo en una solución de $\mathrm{KOH}$, luego puestos en 
alcohol etílico al 70\%, aclarados posteriormente en lactofenol, traspasados enseguida a xilol y finalmente montados en portaobjetos utilizando bálsamo de Canadá. Para la observación y estudio de las preparaciones se utlizaron microscopios Carl Zeis Jena (Alemania) y Reichert (Austria), con cámara clara para dibujo.

Se utilizaron las claves de identificación disponibles (Quate \& Brown, 2004; Tonnoir, 1922 , 1940), además de los aportes de distintos autores que incluyen descripciones y que se señalan para cada especie.

Las abreviaturas utilizadas en el texto corresponden a: $\mathrm{H}, \mathrm{HH}=$ hembra, hembras; $\mathrm{M}$, $\mathrm{MM}=$ macho, machos; $\mathrm{msnm}=$ metros sobre el nivel del mar.

En la lista de especies registradas para Chile, las localidades de Argentina y Chile se detallan por provincias. En el caso de cada especie, se indican los autores que la han mencionado o tratado y, en paréntesis, el género en el cual la han considerado; los autores se citan en orden cronológico y si una referencia no incluye el género, se debe entender que trata a la especie en el género señalado anteriormente por un autor previo.

\section{RESULTADOS}

Lista de especies representadas en la colección MNHNCL (en orden alfabético)

\section{Desmioza edwardsi Tonnoir, 1929}

1 M (preparación), Chile, Región de Los Lagos, Chepu, 19.x.1958, G. Kuschel leg.; corresponde a un ambiente muy húmedo, del tipo selva Valdiviana (Fig. 1).

2 MM (preparaciones), Chile, Región de Los Lagos, Chepu, 15.i.1956, G. Kuschel leg.; selva Valdiviana.

2 MM (preparaciones), Chile, Región de Magallanes, Puerto Williams, Isla Navarino, 21.i.1959, G. Kuschel leg.; bosque subantártico.

2. Desmioza equalis (Tonnoir, 1934) NUEVA COMBINACIÓN

$1 \mathrm{H}$ (preparación), Chile, Región de Magallanes, Puerto Williams, Isla Navarino, 21.i.1959, G. Kuschel leg.; esta localidad se ubica

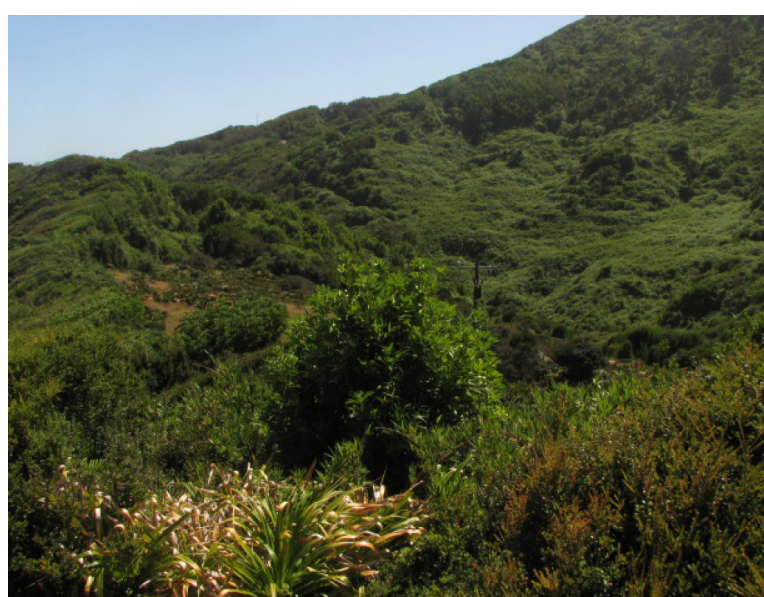

Fig. 1. Vista general del bosque en la costa occidental de Chiloé, cerca de Chepu (Fotografía de Gustavo Novoa).

en la zona del bosque subantártico.

\section{Logima albipennis (Zetterstedt, 1850)}

$1 \mathrm{H}$ (preparación), Chile, Región de Valparaíso, Islas Juan Fernandez, Isla Masafuera (actualmente Isla Alejandro Selkirk), Quebrada Las Casas, 24.i.1955, G. Kuschel leg.; corresponde a un ambiente húmedo (Fig. 2).

$1 \mathrm{H}$ (preparación), Chile, Región Metropolitana, Santiago, Quilicura, 4.xii.1992, M. Elgueta leg; ambiente urbano.

\section{Logima sigma (Kincaid, 1899)}

$1 \mathrm{M}$ (preparación), Chile, Región del Maule, Linares, 11.xii.1992; recolectado en una caja con espárragos frescos, leg. M. Elgueta.

\section{Nemoneura dealbata (Tonnoir, 1929)}

2 MM (preparaciones), Chile, Región de Los Lagos, Frutillar, 15.ii.1956, G. Kuschel leg.; selva Valdiviana.

$1 \mathrm{H}$ (preparación), Chile, Región de Los Lagos, Volcán Calbuco, 200 m a.s.l., 22.ii.1956, G. Kuschel leg.; selva Valdiviana.

3MM, $2 \mathrm{HH}$ (preparaciones), Chile, Región de Magallanes, Isla Welington, Puerto Edén, 400 $\mathrm{m}$ a.s.l., 28.xi.1958, G. Kuschel leg.; bosque subantártico.

6. Nemoneura liparotes Quate \& Brown, 2004

1 M (preparación), Chile, Región de Los Lagos, Chepu, 3.x.1958, G. Kuschel leg.; 


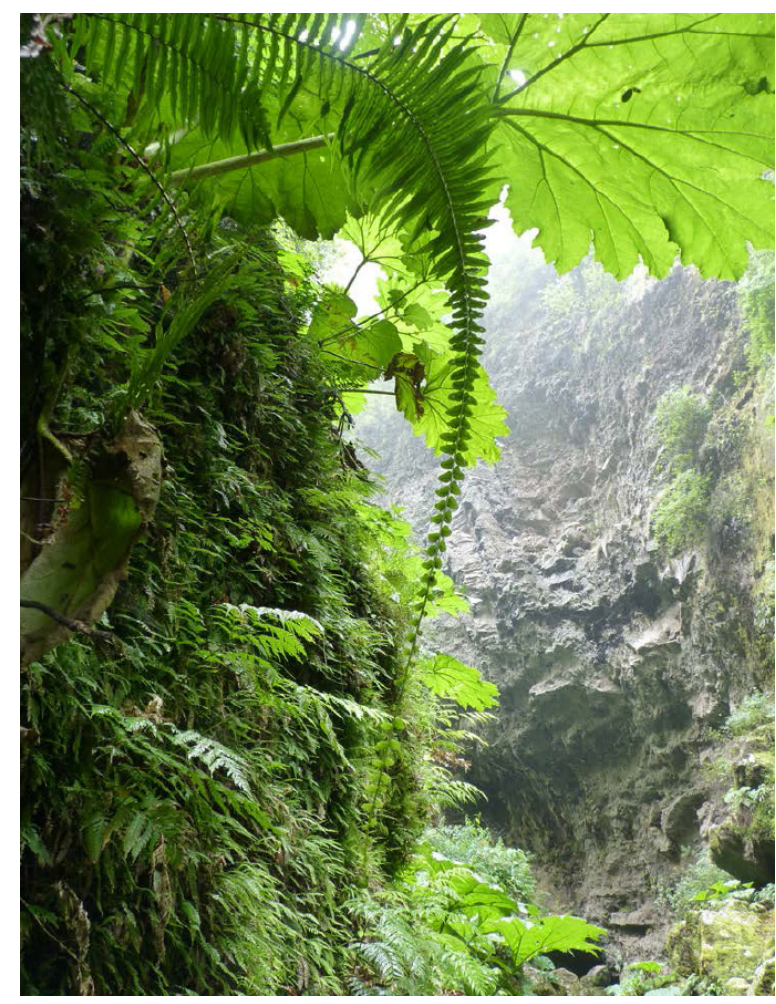

Fig. 2. Aspecto de la Quebrada Las Casas,Isla Alejandro Selkirk, Arhipiélago de Juan Fernández (Fotografía de Patricio Novoa).

ambiente equivalente al de selva Valdiviana.

\section{Psychodocha simillima (Tonnoir, 1929) NUEVA} COMBINACIÓN

5 MM (preparaciones), Chile, Región de Magallanes, Isla Wellington, Puerto Edén, 400 msnm, 3.xii.1958, G. Kuschel leg.; bosque subantártico.

\section{Tinearia alternata (Say, 1825)}

$1 \mathrm{H}$ (preparación), Chile, Región Metropolitana, Santiago Melipilla, xi. 1992; en una caja de frambuesas frescas, leg. M. Elgueta.

$1 \mathrm{H}$ (preparación), Chile, Región del Maule, Linares Linares, xi.1992; ejemplar recolectado en una caja de espárragos frescos, leg. M. Elgueta.

Lista de especies registradas para Chile, en orden alfabético y de acuerdo a subfamilias

\section{Bruchomyiinae}

1. Nemopalpus stuckenbergi Wagner, 2012

Wagner, 2012: 358-360 (In Wagner \&
Stuckenberg, 2012).

Distribución: Chile: Valdivia (Valdivia - Isla Teja).

Comentario: Duckhouse \& Lewis (1989 y nueva version 2007) mencionan a Nemopalpus como error for Nemapalpus. Sabrosky (1999, p. 211) estableció la denominación incorrecta Nemapalpus Macquart, 1838 como grafía original rechazada.

\section{Phlebotominae}

\section{Oligodontomyia isopsi (Léger \& Ferté, 1996)} Lutzomyia isopsi Léger \& Ferté, 1996: 193-195; Dujardin et al. 1999: 736 (Lutzomyia (Oligodontomyia)); Galati et al. 2007: 701 (Oligodontomyia).

Distribución: Chile: Valparaíso ("La Vietacha").

Comentarios: Al describir esta especie, basados en materiales de la ex colección Duret, sus descriptores anotan en una oportunidad (Léger \& Ferté, 1996: 193) Lutzomyia isospi, a pesar de que en el título y en la leyenda de las figuras incluidas se indica claramente Lutzomyia isopsi; por otra parte la derivación del nombre proviene de la denominación de reuniones de especialistas ISOPS (International Symposium on Phlebotomine Sandflies), por lo que el nombre correcto de la especie debe ser el citado en último término.

A pesar de que Léger \& Ferté (1996) citan material examinado de otras dos localidades (Tiltil La Mina y Tregalema, localidad esta última que con seguridad corresponde a la de Tregualemu y que se ubica en la costa sur de la región del Maule), no incluyen esos ejemplares como parte del material tipo ni tampoco lo detallan como material adicional, razón por la cual y en estricto rigor esos ejemplares no pueden ser condiderados como pertenecientes a $O$. isopsi.

Otro problema es respecto de la localidad tipo señalada por los autores como La Vietacha, ya que no existe un lugar ni accidente geográfico con ese nombre en toda el área de la Región de Valparaíso ni tampoco en Chile. El recolector para esa localidad, indicado como Peña en la citada publicación, evidentemente corresponde al entomólogo ya fallecido Luis E. Peña quien mantuvo intercambio con el dipterólogo José 
Pedro Duret; en relación a sus recolecciones se ha consultado al Sr. Alfredo Ugarte, familiar directo de L. Peña, quien señala que los puntos de muestreo de ese material corresponden al cerro La Vizcacha (o cerro Las Vizcachas), que se ubica en el límite norponiente de la provincia de Chacabuco con la provincia de Marga Marga y en aproximadamente $33^{\circ} 5^{\prime} 12.34^{\prime \prime}$ S y $71^{\circ} 1^{\prime} 24.67^{\prime \prime} \mathrm{O}$.

Los datos recopilados sobre Phlebotominae de Chile, indican claramente que se hace necesario efectuar recolecciones sistemáticas, que permitan evaluar la real diversidad del grupo en el país.

El género Oligodontomyia Galati, 1995 junto a Brumptomyia França y Parrot, 1921 integran la subtribu Brumptomyiina, dentro de la tribu Phlebotomini (Galati, 2003, 2007).

\section{Psychodinae}

\section{Desmioza edwardsi (Tonnoir, 1929)}

Pericoma edwardsi Tonnoir, 1929: 12-14; Enderlein, 1937: 97, 99 (Desmioza); Quate, 1963: 183 (Pericoma); Duckhouse, 1973: 9; Quate \& Brown, 2004: 17, 19 (Desmioza); Omad, 2012: 262.

Distribución: Argentina: Neuquén $(23$ km al S de Aluminé, Lago Lacar, Mallín La Heladera, San Martín de los Andes, 7 a $1 \mathrm{~km}$ al W de San Martín de los Andes, $12 \mathrm{~km}$ al S de San Martín de los Andes), Río Negro (Arroyo Blanco, Arroyo La Cantera en Manso Medio, Bariloche, Río Villegas a $57 \mathrm{~km}$ al S de Bariloche), Chubut (Lago Rivadavia en el Parque Nacional Los Alerces, Mallín Lago Rivadavia, Mallín Río Frío). Chile: Llanquihue (Yerbas Buenas), Chiloé (Chepu), Tierra del Fuego (Puerto Williams).

4. Desmioza equalis (Tonnoir, 1934) NUEVA COMBINACIÓN

Pericoma equalis Tonnoir, 1934: 71 (nuevo nombre para Pericoma speciosa Tonnoir, 1929 no Meunier, 1905); Enderlein 1937: 103 (como Thrysocanthus aequalis [sic!]); Duckhouse, 1973: 9 (Pericoma).

= Pericoma speciosa Tonnoir, 1929: 1112; Quate \& Brown, 2004: 19 (Desmioza); Omad, 2012: 262.

Distribución: Argentina: Neuquén (17 km W de Aluminé, $23 \mathrm{~km}$ al S de lago Aluminé, $27 \mathrm{~km}$ al NW de Aluminé, Lago Huechulafquen, Mallín La Heladera, San Martín de los Andes, 7-11 km W de San Martín de los Andes, $12 \mathrm{~km}$ al S de San Martín de los Andes, 81 km NW de San Martín de los Andes), Río Negro (Río Villegas a $57 \mathrm{~km}$ al S de Bariloche), Chubut (Arroyo tributario de Lago Epuyen, Laguna Corcovado, Mallín Lago Rivadavia, Río Frey). Chile: Llanquihue (Casa Pangue), Tierra del Fuego (Puerto Williams).

Comentario: Quate \& Brown (2004) utilizan el nombre Pericoma speciosa Tonnoir, 1929 desconociendo que este es un homónimo posterior de Pericoma speciosa Meunier, 1905, razón por la cual el mismo Tonnoir (1934) había propuesto el nombre de reemplazo Pericoma equalis; por esto y por los cambios propuestos por Quate \& Brown (2004) la denominación correcta para esta especie es Desmioza equalis (Tonnoir, 1934).

\section{Didicrum griseatum (Tonnoir, 1929)}

Pericoma griseata Tonnoir, 1929: 22; Enderlein, 1937: 105 (Didicrum); Quate, 1963: 184-185 (Pericoma); Duckhouse, 1973: 10; Quate \& Brown, 2004: 9-10 (Didicrum).

Distribución: Chile: Chiloé (Ancud).

\section{Didicrum inornatum (Tonnoir, 1929)}

Pericoma inornata Tonnoir, 1929: 23-24; Enderlein, 1937: 102, 105 (Podolepria); Quate, 1963: 185 (Didicrum); Duckhouse, 1973: 10 (Pericoma); Quate \& Brown, 2004: 10 (Didicrum).

Distribución: Argentina: Neuquén (Arroyo Quechuquina, Lago Huechulafquen, 11 km W San Martín de los Andes), Río Negro (Bariloche, Lago Mascardi, Puerto Blest). Chile: Llanquihue (Yerbas Buenas).

\section{Didicrum simplex (Tonnoir, 1929)}

Pericoma simplex Tonnoir, 1929: 25-26; Enderlein, 1937: 105 (Didicrum); Quate, 1963: 184-185 (sinonimia, Pericoma (Didicrum)); Duckhouse, 1973: 10 (Pericoma); Quate \& Brown, 2004: 10-11 (Didicrum); Omad, 2012: 262.

= Mecysmia schoenemanni Enderlein, 1937: 108.

Distribución: Argentina: Neuquén (17 km W de Aluminé, 23 km NW Volcán La Angostura, San Martín de los Andes, 7 a 11 km W de San Martin de los Andes, 81 km NW de San Martin de los 
Andes, Lago Huechulafquen, Mallín La Heladera, Volcán Llanquin $20 \mathrm{~km}$ al S de Confluencia), Río Negro (Bariloche, Lago Mascardi, Mallín La Cortadera, Río Frías), Chubut (Esquel, Lago Puelo, Río Frey, Parque Nacional Los Alerces). Chile: Arauco (Contulmo), Llanquihue (Casa Pangue, Yerbas Buenas).

\section{Didicrum deceptrix Quate \& Brown, 2004}

Didicrum deceptrix Quate \& Brown, 2004: 11. Buenas).

Distribución: Chile: Llanquihue (Yerbas

\section{Didicrum contiguum (Tonnoir, 1929)}

Pericoma contigua Tonnoir, 1929: 2425; Enderlein, 1937: 105 (Didicrum); Quate, 1963: 185; Duckhouse, 1973: 9 (Pericoma); Quate \& Brown, 2004: 11 (Didicrum); Omad, 2012: 262.

Distribución: Argentina: Río Negro (Bariloche, Puerto Blest). Chile: Llanquihue (Casa Pangue).

Comentario: Aunque Quate \& Brown (2004) no citan a esta especie para Chile, Tonnoir (1922) registra un ejemplar de la localidad chilena de Casa Pangue.

10. Didicrum fenestratum (Tonnoir, 1929)

Pericoma fenestrata Tonnoir, 1929: 20; Enderlein, 1937. 92 (Synseodais); Duckhouse, 1973: 10 (Pericoma); Quate \& Brown, 2004: 12 (Didicrum).

Distribución: Chile: Llanquihue (Casa Pangue, Yerbas Buenas).

\section{Didicrum viduatum (Tonnoir, 1929)}

Pericoma viduata Tonnoir, 1929: 26; Enderlein, 1937: 105 (Didicrum viduutum sic!); Duckhouse, 1973: 10 (Pericoma); Quate \& Brown, 2004: 12-13 (Didicrum).

Distribución: Chile: Chiloé (Ancud).

\section{Logima albipennis (Zetterstedt, 1850)}

Psychoda albipennis Zetterstedt, 1850: 3708; Withers, 1988: 75 (Psychoda, sinonimia); Ježek \& Yağci, 2005: 189 (Logima, distribución); Kvifte et al. 2011: 182, 184 (Psychoda (Logima)); Omelková \& Ježek, 2012: 518 (Logima).
= Psychoda severini parthenogenetica Tonnoir, 1940: 53-54; Satchell, 1952: 105; Duckhouse, 1962: 428 (Psychoda parthenogenetica); Duckhouse, 1970: 283; Duckhouse, 1971: 319; Duckhouse, 1973: 12; Duckhouse, 1985: 177; Duckhouse \& Lewis, 1989: 176; Bravo et al. 2006: 12 (Psychoda parthenogenetica).

Distribución: Prácticamente cosmopolita, ya que se encuentra presente en Europa, Rusia, Azores, islas Canarias, Madeira, Turquía, Afganistán, China, India, Japón, Corea, Siria, África, Australia, Nueva Zelanda, isla Campbell, isla Macquarie, islas Georgia del Sur, isla Kerguelen, Región Afrotropical, Estados Unidos de Norteamérica y en América del Sur. Chile: Valparaíso (Islas Juan Fernández, Robinson Crusoe: Cerro Yunque a 915 msnm, Bahía Cumberland; Alejandro Selkirk: al interior de casa, Quebrada las Casas), Santiago (Quilicura).

Hábitat: Sus larvas son saprófagas, por lo que se desarrolla en el estiércol de los animales y en materia vegetal en descomposición, encontrándose comúnmente en casas y establos; los adultos son atraídos a la luz artificial (Satchell, 1952; Omelková \& Ježek, 2012).

Comentario: Logima zetterstedti Ježek, 1983 ha sido considerada como sinónimo de L. albipennis, pero esta sinonimia sólo es parcial; el aclarar y resolver definitivamente esto, requiere del estudio del material tipo de Psychoda albipennis Zetterstedt, 1850.

\section{Logima sigma (Kincaid, 1899)}

Psychoda sigma Kincaid, 1899: 31-32; Del Rosario, 1936: 114-116; Duckhouse, 1966: 211 ; Duckhouse \& Lewis, 1989: 177; Withers, 1988: 72 (sinonimia); Ježek, 2003: 125 (Logima); Kvifte et al. 2011: 182.

= Psychoda surcoufi Tonnoir, 1922: 74-76; Satchell, 1947: 53-54; Duckhouse, 1966: 211 213; Duckhouse, 1971: 318, 324; Duckhouse, 1973: 13; Duckhouse, 1976: 47; Duckhouse, 1985: 176; Withers, 1988: 72 (sinonimia); Duckhouse \& Lewis, 1989: 177; Bravo et al. 2006: 12 (Psychoda (Logima)).

= Psychoda subinmaculata Tonnoir, 1929:

6-7; Enderlein, 1937: 86. = Psychoda spatulata Satchell, 1950: 166. 
Distribución: De amplia distribución, presentándose en Europa, Australia, Nueva Zelanda, isla Macquarie, isla Campbell, islas Auckland, isla Antipodas, Santa Helena, Estados Unidos de Norteamérica, Hawaii. Chile: Linares (Linares), Llanquihue (Puerto Montt), Chiloé (Ancud).

Hábitat: Se encuentra en jardines, existiendo registros como afectando papas y también ha sido criada de estiércol de vacuno (Withers 1988).

Nota: Withers (1988: 72) considera a Psychoda surcoufi Tonnoir (1922) como nombre válido y como sinónimo a Psychoda sigma Kincaid (1899); esto en realidad es a la inversa, ya que el nombre Psychoda sigma tiene prioridad sobre Psychoda surcoufi (ver Del Rosario 1936 y Ježek 2003)

\section{Nemoneura punctata (Philippi, 1865)}

Psychoda punctata Philippi, 1865: 631; Tonnoir, 1929: 27-29 (Nemoneura); Enderlein, 1937: 101, 107; Duckhouse, 1973: 8; Quate \& Brown, 2004: 111, 112.

Distribución: Chile: Valdivia (sin localidad precisa), LLanquihue (Peulla), Chiloé (isla de Chiloé sin localidad precisa).

\section{Nemoneura dealbata (Tonnoir, 1929)}

Nemoneura dealbata Tonnoir, 1929: 29; Enderlein, 1937: 107; Duckhouse, 1973: 8; Quate \& Brown, 2004: 113; Omad, 2012: 262.

Distribución: Argentina: Neuquén (Arroyo Quechuquina, Lago Huechulafquen, Puerto Blest, San Martín de los Andes, 7 a 11 km W de San Martín de los Andes), Chubut (Río Frey, Parque Nacional Los Alerces). Chile: Llanquihue (Casa Pangue, Ensenada, Frutillar, Volcán Calbuco, Volcán Osorno sector La Angostura), Chiloé (isla de Chiloé sin localidad precisa), Última Esperanza (Puerto Edén).

16. Nemoneura liparotes Quate \& Brown, 2004 Nemoneura liparotes Quate \& Brown, 2004: 113, 115. (Chepu).

Chile: Llanquihue (Yerbas Buenas), Chiloé

17. Pericoma chilensis (Tonnoir, 1929)

Pericoma chilensis Tonnoir, 1929: 14-15;
Enderlein, 1937: 96, 99 (Synmormia); Duckhouse, 1973: 9 (Pericoma).

Distribución: Chile: Chiloé (Ancud).

Comentario: La exacta posición sistemática de esta especie no ha sido aclarada.

18. Pericoma niveopunctata Tonnoir, 1929

Pericoma niveopunctata Tonnoir, 1929: 18-20; Enderlein, 1937: 105 (Didicrum niveopunctatum); Quate, 1963: 185 (Pericoma); Duckhouse, 1973: 10 (Pericoma).

Distribución: Chile: Chiloé (Castro)

Comentario: Sólo se conoce la hembra de esta especie y las figuras resultan insuficientes, como para poder aclarar su correcta posición sistemática.

19. Pericoma squamitarsis Tonnoir, 1929

Pericoma squamitarsis Tonnoir, 1929: 21;

Duckhouse, 1973: 10.

Distribución: Chile: Llanquihue (Casa Pangue).

Comentario: Especie hasta ahora no reconocida.

20. Psychoda dubia Bigot, 1888

Psychoda dubia Bigot, 1888: 4; Tonnoir, 1929: 4; Duckhouse, 1973: 15.

Distribución: Chile: Magallanes (Bahía Orange en Tierra del Fuego).

Comentario: Hasta ahora no se ha podido reconocer a esta especie.

21. Psychoda fimbriatissima Blanchard, 1852

Psychoda fimbriatissima Blanchard, 1852: 351; Philippi, 1865: 631; Tonnoir, 1929: 4; Duckhouse, 1973: 16.

Distribución: Chile: Coquimbo (Coquimbo), Santiago (Santiago), Valdivia (sin localidad precisa).

Comentario: No ha sido reconocida esta especie en colecciones.

22. Psychoda hyalinata Blanchard, 1852

Psychoda hyalinata Blanchard, 1852: 351-

352; Tonnoir, 1929: 4; Duckhouse, 1973: 16.

Distribución: Chile (sin localidad precisa).

Comentario: Esta especie permanece hasta ahora sin ser identificada en colecciones. 
23. Psychoda masatierrensis Satchell, 1952

Psychoda masatierrensis Satchell, 1952: 106-107; Duckhouse, 1973: 12; Bravo et al. 2006: 12 .

Distribución: Chile: Islas Juan Fernández (Robinson Crusoe: Bahía Cumberland, El Camote a 400 msnm; Alejandro Selkirk: en establos, en casa habitación).

Comentario: La correcta asignación a género no ha sido aclarada hasta ahora.

\section{Psychoda minutissima Enderlein, 1940}

Psychoda (Threticus) minutissima

Enderlein, 1940: 673; Duckhouse, 1973: 16 (Psychoda).

Distribución: Chile: Islas Juan Fernández (Robinson Crusoe).

Comentario: Especie hasta ahora no reconocida en colecciones.

\section{Psychoda notata Blanchard, 1852}

Psychoda notata Blanchard, 1852: 351; Tonnoir, 1929: 4; Duckhouse, 1973: 16.

Distribución: Chile (sin localidad precisa).

Comentario: No reconocida en colecciones.

26. Psychoda pulla Rondani, 1864

Psychoda pulla Rondani, 1864: 90; Tonnoir, 1929: 4; Duckhouse, 1973: 16.

Distribución: Chile (sin localidad precisa)
Comentario: Especie no representada en colecciones y su descripción no permite su reconocimiento.

\section{Psychoda tenella Philippi, 1865}

Psychoda tenella Philippi, 1865: 632;

Tonnoir, 1929: 4; Duckhouse, 1973: 16.

Distribución: Chile: Santiago (Santiago)

Comentario: Especie hasta ahora no reconocida en colecciones y no existen ejemplares en la colección del MNHNCL, por lo que su eventual reconocimiento en material de colección resulta aún más problemático.

28. Psychodocha cinerea (Banks, 1894) (Fig. 3)

Psychoda cinerea Banks, 1894: 331;

Satchell, 1947: 56-57; Satchell, 1952: 105;

Duckhouse, 1966: 208-209; Duckhouse, 1973: 12; Ježek, 1984: 136 (Psychodocha); Withers, 1988: 70-71 (Psychoda, sinonimia); Duckhouse \& Lewis, 1989: 175; Ježek \& Yağci, 2005: 190 (Psychodocha, distribución); Bravo et al. 2006: 11 (Psychoda (Psychodocha)); Kvifte et al. 2011: 182, 185; Omelková \& Ježek, 2012: 524 (Psychodocha).

Distribución: Cosmopolita. Europa,

Turquía, Argelia, Israel, Túnez, Afganistán, Irán, islas Azores, islas Canarias, Madeira, África meridional, Australia, Nueva Zelanda, Estados Unidos de Norteamérica, Puerto Rico, Argentina, Brasil, Canadá. Chile: Valparaíso (Islas Juan

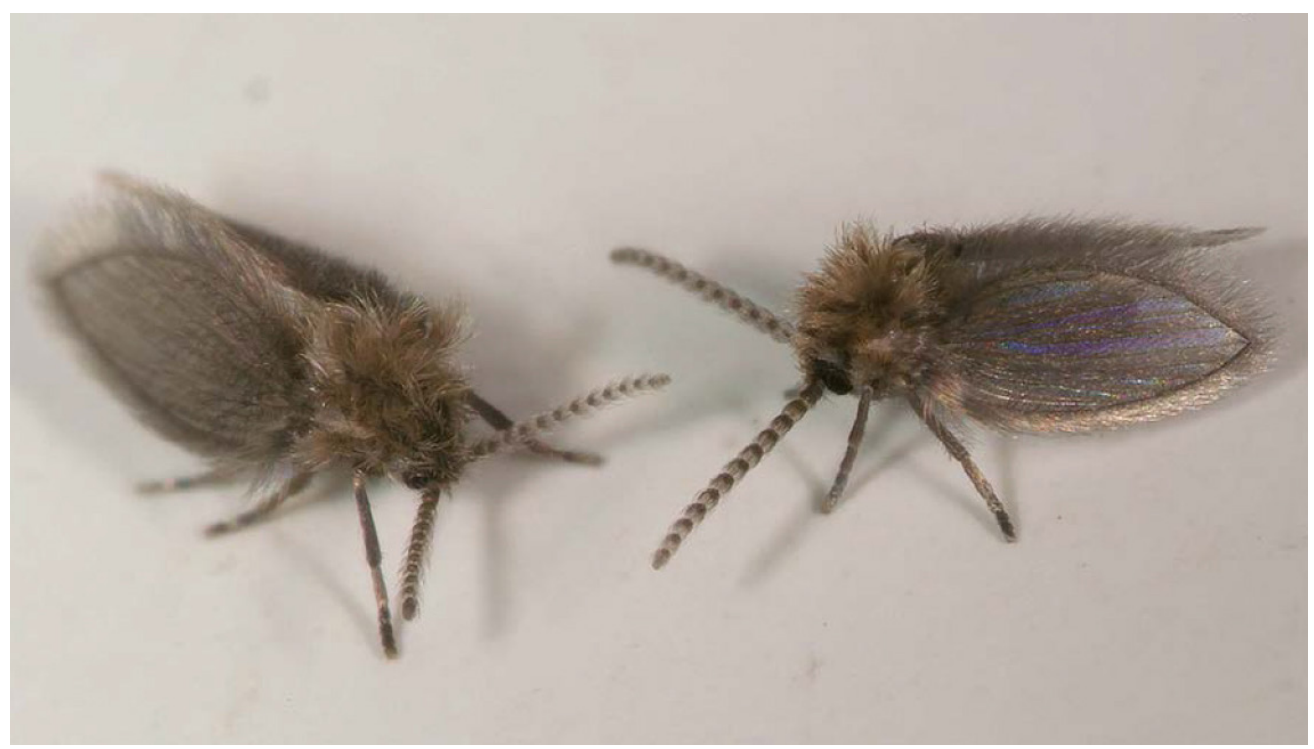

Fig. 3. Adultos de Psychodocha cinerea en baño de casa (Brno, Czech Republic; fotografía de Miroslav Kopeček). 
Fernández,(Robinson Crusoe: Bahía Cumberland); Santiago (Santiago).

Hábitat: Sus larvas son saprófagas y se desarrollan en materia orgánica, asociándose ocasionalmente a hongos; los adultos (Figura 3) se encuentran frecuentemente en baños con poca limpieza, criándose en desagües y sectores en que estos gotean. Es una especie asociada ambientes urbanos (Satchell 1952; Withers, 1988; Omelková \& Ježek, 2012).

29. Psychodocha pseudocompar (Tonnoir, 1929) NUEVA COMBINACIÓN

Psychoda (Threticus) pseudocompar Tonnoir, 1929: 7-8; Enderlein, 1937: 87 (Threticus, Chile); Duckhouse, 1973: 13 (Psychoda); Bravo et al. 2006: 11 (Psychoda (Psychodocha), Chile).

Distribución: Argentina: Neuquén (Lago Correntoso). Chile: (sin localidad precisa).

Comentario: Esta especie no fue mencionada por Ježek (1984).

30. Psychodocha simillima (Tonnoir, 1929) NUEVA COMBINACIÓN

Psychoda (Threticus) simillima Tonnoir, 1929: 8-9; Enderlein, 1937: 87 (Threticus); Duckhouse, 1973: 13 (Psychoda); Bravo et al. 2006: 11 (Psychoda (Psychodocha)); Cordeiro et al. 2013: 135-137 (Psychoda); Omad, 2012: 262; Omad et al. 2013: 402.

Distribución: Argentina: Neuquén (Bariloche), Chubut (El Hoyo, Puerto Patriada, 42 $\left.{ }^{\circ} 29^{\prime} 44^{\prime \prime} \mathrm{S}-71^{\circ} 23^{\prime} 21^{\prime \prime} \mathrm{W}\right)$. Chile: Última Esperanza (Puerto Edén), Magallanes (Península Muñoz Gamero).

Nota: Bravo et al. (2006) citan a esta especie como encontrada anteriormente en "P. de los libres" y señalan como referencia a Tonnoir (1929) lo cual es un error, ya que este último autor sólo la señala de Bariloche, en la provincia de Río Negro.

Comentario: Esta especie no fue mencionada por Ježek (1984).

\section{Syntomolaba complicata (Tonnoir, 1929)}

Pericoma complicata Tonnoir, 1929: 1516; Enderlein, 1937: 97, 100 (Syntomolaba, Chile); Duckhouse, 1973: 9 (Pericoma); Quate \&
Brown, 2004: 115 (Syntomolaba).

Distribución: Argentina: Río Negro (Puerto Blest). Chile (sin localidad precisa).

Comentario: La posición sistemática correcta de esta especie necesita ser aclarada.

32. Tinearia alternata (Say, 1825)

Psychoda alternata Say, 1825: 358; Banks, 1894: 330; Duckhouse, 1973: 12; Ježek, 1977: 232 (Tinearia); Withers, 1988: 69-7(Psychoda); Ježek, 1984: 141 (Tinearia); Duckhouse \& Lewis, 1989: 175 (Psychoda); Ježek \& Yağci, 2005: 190 (Tinearia, distribución); Bravo et al. 2006: 5, 11 (Psychoda (Tinearia); Ježek et al. 2011: 191, 192 (Tinearia); Kvifte et al. 2011: 182, 185 (Psychoda (Tinearia); Omad, 2012: 262 (Psychoda); Omelková \& Ježek, 2012: 518 (Tinearia).

= Psychoda septempunctata Philippi, 1865: 631; Tonnoir, 1934: 79-80; Duckhouse, 1973: 16; Withers, 1988: 69 (como Psychoda "sexpunctata" error!).

Distribución: Cosmopolita. Europa, Rusia, Turquía, Afganistán, Bangladesh, Borneo, India, Japón, Malasia, Mongolia, Corea del Norte, Filipinas, Samoa, Taiwan, África, Australia, Nueva Zelanda, Costa Rica, Nicaragua, Panamá, Jamaica, América del Norte, América del Sur. Argentina: Chubut (Laguna Terraplen, Río Carrileufú, $42^{\circ} 29^{\circ}$ $\left.44^{\prime \prime} \mathrm{S}-71^{\circ} 23^{\prime} 21^{\prime \prime} \mathrm{W}\right)$. Chile: Melipilla (Melipilla), Santiago (Santiago), Linares (Linares).

Hábitat: Sus larvas se desarrollan en cualquier condición en que exista materia orgánica, incluyendo excrementos, y también se asocian a hongos; en ciertas condiciones y al presentarse en grandes cantidades, puede llegar a afectar algunas operaciones en el tratamiento de aguas. Sus larvas han sido encontradas también en urinarios, pozos sépticos y desagües (Satchell 1947, Withers 1988, Omelková \& Ježek 2012).

Comentario: Withers (1988) detalla la sinonimia de esta especie e incluye a Psychoda „sexpunctata" Philippi, 1865; esto es un error, ya que el nombre correcto es Psychoda septempunctata Philippi, 1865.

\section{Sycoracinae}

33. Aposycorax chilensis (Tonnoir, 1929)

Sycorax chilensis Tonnoir, 1929: 31-32; 
Enderlein, 1937: 109; Duckhouse, 1972: 233235 (Aposycorax); Duckhouse, 1973: 6 (Sycorax); Ježek, 1999: 84; Bravo, 2003: 385, 386; Azar et al. 2007: 7 (Aposycorax); Bejarano et al. 2008: 30 (Sycorax); Petrulevičius et al. 2011: 92 (Aposycorax).

Distribución: Chile: Llanquihue (Casa Pangue, Peulla), Chiloé (Ancud).

Comentario: Ježek (1999) no considera valido el género Aposycorax Duckhouse, 1972.

\section{Trichomyiinae}

34. Trichomyia aurea Duckhouse, 1972

Trichomyia aurea Duckhouse, 1972: 241243, 245; Omad, 2012: 263.

Distribución: Argentina: Neuquén (Mallín La Heladera). Chile: Chiloé (Ancud, Chacao, Chepu, Degañ, Puntra)

35. Trichomyia capitanea Duckhouse, 1972 Trichomyia capitanea Duckhouse, 1972: 247-248.

Distribución: Chile: Chiloé (Chepu).

36. Trichomyia chepuensis Duckhouse, 1972

Trichomyia chepuensis Duckhouse, 1972: 248, 250.

Distribución: Chile: Chiloé (Chepu).

\section{Trichomyia edwardsi Tonnoir, 1929}

Trichomyia edwardsi Tonnoir, 1929:

30-31; Enderlein, 1937: 111 (Chile); Satchell, 1956: 151; Duckhouse, 1972: 240, 260; Duckhouse, 1973: 5.

Distribución: Argentina: Río Negro (Puerto Blest). Chile: Llanquihue (Casa Pangue).

Nota: Duckhouse $(1972,1973)$ manifiesta dudas acerca de la identificación del ejemplar de Casa Pangue que Satchell (1956) adscribe a esta especie.

38. Trichomyia figuieroai Duckhouse, 1972

Trichomyia figuieroai Duckhouse, 1972: 256, 258. error!).

Omad, 2012: 263 (como T. figueroai,

Distribución: Argentina: Río Negro Arroyo Blest, Mallín los Patos). Chile: Chiloé (Chepu,
Degañ).

39. Trichomyia kenricki Duckhouse, 1972

Trichomyia kenricki Duckhouse, 1972: 254, 256.

Distribución: Chile: Chiloé (Ancud).

40. Trichomyia manni Duckhouse, 1972

Trichomyia manni Duckhouse, 1972: 250, 252, 254.

Distribución: Chile: Chiloé (Ancud, Chepu, Degañ).

Otras menciones para Chile, sin identificación precisa

Existen otras citas de la presencia de representantes de Psychodidae en Chile, pero sin mayor identificación; estas corresponden a:

Psychoda sp., de Valparaíso (Islas Juan Fernández - Robinson Crusoe), de acuerdo a Satchell (1952, p. 106).

Psychoda sp., de Malleco (Angol), señalada por Duckhouse (1973, p. 13).

Phlebotominae sp., de Chile y sin localidad precisa como lo indica Le Pont et al. (1988, p. 166); ahora se conoce que esta identificación se fundamento en un ejemplar recolectado en la costa central de Chile (Algarrobo, en la provincia de San Antonio), tal como se aclara en la Instroducción de este aporte.

Lutzomyia sp., de Parinacota (Putre), como lo informa González (2013, p. 4).

\section{AGRADECIMIENTOS}

Especialmente al Dr. Guillermo "Willy" Kuschel, conocido especialista en Curculionoidea (Coleoptera), nacido en Chile pero radicado desde aproximadamente 1960 en Nueva Zelanda, en donde trabajó en el Landcare Research (previamente DSIR) hasta unos pocos años atrás. Durante su permanencia en Chile trabajó en la Universidad de Chile y efectuó recolecciones de insectos a lo largo y ancho de todo el país; algunas de sus variadas, intensivas y sistemáticas recolectas de insectos, se encuentran depositadas en el Museo Nacional de Historia Natural (Santiago, Chile).

A Patricio Novoa (Jardín Botánico Nacional, 
Viña del Mar) y Gustavo Novoa (Chiloé) por su desinteresada colaboración al proporcionarnos fotografías de ambientes, de localidades citadas en este trabajo. Del mismo modo agradecemos a Miroslav Kopeček (Brno, Czech Republic) por proporcionarnos fotografías de adultos de Psychodocha cinerea.

A Eunice A. B. Galati por el envío de literatura. A Francois Le Pont por compartir antecedentes sobre la identificación de muestras de Phlebotominae de Chile.

A Petr Kment (National Museum, Praha, Czech Republic) por su revisión del manuscrito.

Este estudio ha sido parcialmente financiado por el Ministry of Culture - Czech Republic (DKRVO 2014/13, National Museum, 0002327201).

\section{LITERATURA CITADA}

Azar, D., Tahchy A. \& Perrichot, V. (2007). The oldest Sycoracinae (Diptera: Psychodidae) from the French Cretaceous amber. Alavesia, 1: 5-10.

Banks, N. (1894). Some Psychodidae from Long Island, N. Y. The Canadian Entomologist, 26: 329-332.

Bejarano, E., P. Duque \& Vélez, I. D. (2008). A new species of Sycorax (Diptera, Psychodidae, Sycoracinae) from the Pacific coast of Columbia. Revista Brasileira de Entomologia, 52(1), 28-31.

Bigot, J. F. M. (1888). Diptères. In: Ministéres de la Marine et de l'Instruction Publique (eds.), Mission Scientifique du Cap Horn 1882-1883, Insectes, 6(2), 1-45, 4 lams. Gauthier-Villars et fils Imprimeurs-Libraires, Paris.

Blanchard, E. (1852). In: C. Gay (ed.), Historia fisica y politica de Chile, Zoologia, 7, 351. Imprenta de Maulde et Renou, Paris.

Bravo, F., D. Cordeiro \& Chagas, C. (2006). Two new species and new records of Psychoda Latreille (Diptera: Psychodidae: Psychodinae) from Brazil, with comments on supraspecific classification of the genus. Zootaxa, 1298, 1-15.

Cordeiro, D., G. Omad, F. Bravo \& de Carvalho, J. B. (2013). New records, and the first description of a female, for Psychoda simillima (Diptera: Psychodidae) in southern South America. Iheringia, Série Zoologia, 103(2), 134-137.

del Rosario, F. (1936). The American species of Psychoda (Diptera: Psychodidae). Philippine Journal of Science, 59, 85-148. Donoso-Barros, R. \& Cambiazo, C. C. (1950). Un caso de myasis digestiva por larvas de Psychoda sp. Revista Médica de Chile, 78, 471.

Duckhouse, D. A. (1962). Some British Psychodidae

(Diptera, Nematocera): descriptions of species and a discussion on the problem of species pairs. Transactions of the Royal Entomological Society of London, 114: 403-436.

Duckhouse, D. A. (1966). Psychodidae (Diptera, Nematocera) of Southern Australia: subfamily Psychodinae. Transactions of the Royal Entomological Society of London, 118, 153-220.

Duckhouse, D. A. (1970). Diptera: Psychodidae of South Georgia. Pacific Insects Monograph, 23, 283.

Duckhouse, D. A. (1971). Entomology of the Aucklands and other island South of New Zealand: Diptera: Psychodidae. Pacific Insects Monograph, 27, 317-325.

Duckhouse, D. A. (1972). Psychodidae (Diptera, Nematocera) of South Chile, subfamilies Sycoracinae and Trichomyinae. Transactions of the Royal Entomological Society of London, 124(3), 231-268.

Duckhouse, D. A. (1973). A catalogue of the Diptera of the Americas south of the United States. 6A. Family Psychodidae, subfamilies Bruchomyinae, Trichomyiinae, Sycoracinae and Psychodinae. Universidade de Sao Paulo, Museu de Zoologia. 29 p.

Duckhouse, D. A. (1976). 9. Fam. Psychodidae. La faune terrestre de l'île de Sainte - Hélené (3). Musee Royal de l'Afrique Centrale, Annales, Sciences Zoologiques, 215, 4648.

Duckhouse, D. A. (1977) Psychodidae. In: S. H. Hurlbert (ed.), Biota acuática de Sudamérica Austral. San Diego State University, San Diego. XIV + 342 pp.

Duckhouse, D. A. (1985). Psychodidae (Diptera, 
Nematocera) of the subantarctic islands, with observations on the incidence of parthenogenesis. International Journal of Entomology, 27(3), 173-184.

Duckhouse, D. A. \& Lewis, D. J. 1(989). Superfamily Psychodoidea 15. Family Psychodidae. Pp. 166-179. In: N. L: Evenhuis (ed.), Catalog of the Diptera of the Australasian and Oceanian Regions. Bishop Museum Press, Honolulu and E. J. Brill, Leiden. 1155 p.

Enderlein, G. (1937). Klassifikation der Psychodiden (Dipt.). Deutsche Entomologische Zeitschrift, 4, 81-112.

Enderlein, G. (1940). Die Dipterenfauna der Juan Fernandez-Inseln und der Oster-Insel. In: C. Skottsberg (ed.), The Natural History of Juan Fernandez and Easter Island, 3, 673. Almqvist \& Wiksells Bocktrickery A.B., Uppsala.

Foote, B. A. (1991). Psychodidae (Psychodoidea). In: F. W. Stehr (ed.), Inmature insects, 2: 746. Kendal / Hunt Publishing Company, Dubuque.

Galati, E. A. B. (2003). Morfologia, terminologia de adultos e identificação dos táxons da América. Pp. 53-175. In: EF Rangel, R Lainson (eds.), Flebotomíneos do Brasil, Fiocruz, Rio de Janeiro.

Galati, E. A. B., dos Santos, D. R. \& Da Silva, A. M. (2007). Brumptomyia angelae, a new species of Phlebotominae (Diptera, Psychodidae) of the Atlantic forest of the state of Paraná, Brazil. Memórias do Instituto Oswaldo Cruz, 102(6), 701-705.

González, C. R. (2013). Reporte de Lutzomyia (Diptera: Psychodidae) en Chile. Parasitología al día, Resúmenes Reuniones Científicas, 4, 3.

Ježek, J. (1983). Contribution to the taxonomy of genus Logima Eat. (Diptera, Psychodidae). Acta Entomologica Musei Nationalis Pragae, 41, 213-234.

Ježek, J. (1984). Six new genera of the tribe Psychodini End. (Diptera, Psychodidae). Acta Faunistica Entomologica Musei Nationalis Pragae, 17, 133 - 153.

Ježek, J. (1999). Comments on the correct grammatic gender of Sycorax Curt. and
Philosepedon Eat. (Diptera: Psychodidae) with world catalogue. Dipterologica bohemoslovaca, 9, 83-87.

Ježek, J. (2003). New faunistic data and check-list of non Phlebotomine moth flies (Diptera, Psychodidae) from the Czech and Slovak Republics. Časopis Národního Muzea, Řada prírodovědná, 172, 121-132.

Ježek, J. \& Yağci, S. (2005). Common non-biting moth flies (Insecta, Diptera, Psychodidae) new to the fauna of Turkey. Acta Parasitologica Turcica, 29(3), 188-192.

Ježek, J., F. Le Pont, E. Martínez \& Mollinedo, S. (2011). Three new species of non-biting moth flies (Diptera: Psychodidae: Psychodinae) from Bolivia, with notes on higher taxa of the subfamily. Acta Entomologica Musei Nationalis Pragae, 51(1): 183-210.

Kincaid, F. (1899). The Psychodidae of the Pacific coast. Entomological News, 10: 30-37.

Kvifte, G. M., Ø. Håland \& Andersen, T. (2011). A revised checklist of Norwegian moth flies (Diptera, Psychodidae). Norwegian Journal of Entomology, 58, 180-188.

Le Pont, F., E. Martínez, J.-M. Torrez-Espejo \& Dujardin, J.-P. (1998). Phlébotomes de Bolivie: description de cinq nouvelles espèces de Lutzomyia de la región subandine (diptera, Psychodidae). Bulletin de la Société entomologique de France, 103(2), 159-173.

Léger, N. \& Ferté, H. (1996). Première mention de Phlébotomes au Chili et description de Lutzomyia isopsi n. sp. (DipteraPsychodidae). Parasite, 3, 193-195.

Mariluis, J. C., P. R.Mulieri, L. D. Patitucci \& Oliva, A. (2007). Cystomyiasis by larvae of a Psychoda sp. (Diptera: Psychodidae): first case for Argentina. Canadian Society of Forensic Science Journal, 40(4), 187-188.

Meunier, F. (1905). Monographie des Psychodidae de l'ambre de la Baltique. Annales Musei Nationalis Hungarici, 3, 235-255.

Moya-Arévalo, R. H., S. Ibáñez-Bernal \& SuárzLanda, M. T. (2012). First record of Didicrum Enderlein (Diptera: Psychodidae, Psychodinae) from the Northern Andes bioregion, with the description of a new species. Zootaxa, 3577, 80-88. 
Omad, G. H. (2012). Inventario preliminar y nuevos registros de Psychodidae no Phlebotominae (Diptera, Nematocera) para Argentina, con especial referencia a la Patagonia. Revista de la Sociedad Entomológica Argentina, 71(3-4), 257-264.

Omad, G. (2014). Two new species of Didicrum Enderlein (Diptera, Psychodidae, Psychodinae) from Argentinean Patagonia. Zootaxa, 3794(4), 565-574.

Omad, G. \& Rossi, G. C. (2012). A new species and records of Alepia Enderlein (Diptera, Psychodidae, Psychodinae) from Argentina. Zootaxa, 3397, 62-68.

Omad, G. H., C. Mangudo, D. Anjos-Santos \& Gleiser, R. M. (2013). Descriptions of pupae of three Psychodinae species (Diptera: Psychodidae) from Argentina. Annales Zoologici, 63(3), 401-408.

Omelková, M. \& Ježek, J. (2012). A new species of the genus Trichomyia (Diptera: Psychodidae) and new faunistic data on nonphlebotomine moth flies from the Podyji NP and its surroundings (Czech Republic). Acta Entomologica Musei Nationalis Pragae, 52(2), 505-533.

Petrulevičius, J. F., Nel, A., De Franceschi, D., Goillot, C., Antoine, P.-O., Salas-Gismondi, R \& Flynn, J. J. (2011). First fossil blood sucking Psychodidae in South America: a sycoracine moth fly (Insecta: Diptera) in the middle Miocene Amazonian amber. Insect Systematics \& Evolution, 42, 87-96.

Philippi, R. A. (1865). Aufzählung der chilenischen Dipteren. Verhandlungen der KaiserlichKöniglichen Zoologisch-Botanischen Gesellschaft in Wien, 15, 595-782, lams. XXIV-XXVIII.

Quate, L. W. (1963). Review of G. Enderlein's non holarctic genera of Psychodidae and description of a new species (Diptera). Transactions of the Royal Entomological Society of London, 115(6), 181-196.

Quate, L. W. \& Brown, B. V. (2004). Revision of Neotropical Setomimini (Diptera: Psychodidae: Psychodinae). Contributions in Science, 500, 1-117.

Rondani, C. (1864). Dipterorum species et genera aliqua exotica revisa et annotata, novis, nonullis descriptis. Archivio per la zoologia, l'anatomia e la fisiologia, 3, 1-99, 1 lam.

Sabrosky, W. C. (1999). Family - Group Names in Diptera and Bibliography. Myia, North American Dipterist's Society. Backhyus Publishers, Leiden, 10, 584 pp.

Satchell, G. H. (1947). The larvae of the British species of Psychoda (Diptera: Psychodidae). Parasitology, 38, 51-69.

Satchell, G. H. (1950). The New Zealand Psychodidae: A study based upon the collection and manuscript notes of the late Dr. A. L. Tonnoir. The Transactions of the Royal Entomological Society of London, 101(5), 147-178.

Satchell, G. H. (1952). Los insectos de las Islas Juan Fernandez 8. Psychodidae (Diptera). Revista Chilena de Entomología, 2, 105107.

Satchell, G. H. (1956). On the genus Trichomyia Haliday (Diptera, Psychodidae), with descriptions of five new species. Proceedings of the Royal Entomological Society of London, (B) 25, 147-156.

Say, T. (1825). Appendix. Part I.- Natural History. 1. Zoology. Pp. 253-378. In: W. H. Keating (ed.), Narrative of an expedition to the source of St. Peter's river, Lake Winnepeek, Lake of the Woods, \&c. \&c. performed in the year 1823, by order of the Hon. J. C. Calhoun, Secretary of War, under the command of Stephen H. Long, Major U. S. T. E. Volume 2, 459 p. H. C. Carey \& I. Lea, Philadelphia. (1824).

Stuardo, C. S. (1946). Catálogo de los dípteros de Chile. Ministerio de Agricultura, Dirección General de Agricultura, Santiago. 253 p.

Tonnoir, A. L. (1922). Synopsis des espèces européenes du genre Psychoda (Diptères). Annales de la Societé Entomologique de Belgique, 62, 49-88.

Tonnoir, A. L. (1929). Diptera of Patagonia and South Chile, pt.1. Fascicle I-Psychodidae. British Museum (Natural History), London. 32 pp.

Tonnoir, A. L. (1934). Notes synonymiques sur quelques Psychodidae (Diptera). Bulletin \& Annales de la Societé Rroyale 
dÉntomologie de Belgique, 74, 69-82.

Tonnoir, A. L. (1940). A synopsis of the British Psychodidae (Dipt.) with descriptions of new species. Transactions of the Society for British Entomology, 7, 21-64.

Wagner, R. (2012). Ver Wagner, R. \& B. R. Stuckenberg.

Wagner, R. \& Stuckenberg, B. R. (2012). New fossil and extant species of Nemopalpus Macquart (Diptera: Psychodidae: Bruchomyinae). African Invertebrates, 53(1), 355-367.

Withers, P. (1988). Revisionary notes on British species of Psychoda Latreille (Diptera,
Psychodidae) including new synonyms and a species new to science. British Journal of Entomology and Natural History, 1, 6976.

Young, D. G. \& Duncan, M. A. (1994). Guide to the identification and geographic distribution of Lutzomyia sand flies in Mexico, the West Indies, Central and South America (Diptera: Psychodidae). Memoirs of the American Entomological Institute, 54, 1-881.

Zetterstedt, J. W. (1850). Diptera scandinaviae disposita et descripta. Lundae 9: 33673710. 